\title{
MINIMAL SEQUENCES IN SEMIGROUPS
}

\author{
BY \\ MOHAN S. PUTCHA (1)
}

\begin{abstract}
In this paper we generalize a result of Tamura on S-indecomposable semigroups. Based on this, the concept of a minimal sequence between two points, and from a point to another, is introduced. The relationship between two minimal sequences between the same points is studied. The rank of a semigroup $S$ is defined to be the supremum of the lengths of the minimal sequences between points in $S$. The semirank of a semigroup $S$ is defined to be the supremum of the lengths of the minimal sequences from a point to another in $\boldsymbol{S}$. Rank and semirank are further studied.
\end{abstract}

Introduction. Semilattice decompositions of semigroups were first defined and studied by Clifford [1]. Since then several people have worked on this topic, notably Tamura [5] [9]. The author's work on the subject can be found in [3], [4]. In this paper, we start by generalizing a result of Tamura [8] (or [9]) on $\delta$ indecomposable semigroups. Based on this, the concept of a minimal sequence between two points, and from a point to another, is introduced. The relationship between two minimal sequences between the same points is studied. The rank of a semigroup is defined to be the supremum of the lengths of the minimal sequences between points in the semigroup. The semirank of a semigroup is defined to be the supremum of the lengths of the minimal sequences from a point to another in the semigroup. Rank and semirank are further studied. To understand this paper, the reader need only be aware of the first few chapters of Clifford and Preston [2] and Tamura's decomposition theorem. (See any of [5], [6], [8] or [9]. It was rediscovered by Petrich [10].)

1 Preliminaries. Throughout, $S$ will denote a semigroup and $Z^{+}$the set of positive integers. A congruence $\sigma$ on $S$ is called a semilattice congruence if $S / \sigma$ is a semilattice. $S \times S$ is the universal congruence on $S$. $S$ is $\delta$-indecomposable if $S \times S$ is the only semilattice congruence on $S$.

Definition. Let $a, b \in S$. Then

(1) $a \mid b$ if and only if $b \in S^{1} a S^{1}$. | is transitive and reflexive.

(2) $\rightarrow$ is defined as $a \rightarrow b$ iff $a \mid b^{i}$ for some $i \in Z^{+}$; let $\rightarrow^{0}$ denote $\rightarrow$, i.e., $\rightarrow^{0}=\rightarrow$.

(3) $a \rightarrow^{n+1} b$ iff there exists $x \in S$ such that $a \rightarrow^{n} x \rightarrow b$.

(4) $a \rightarrow^{\infty} b$ iff $a \rightarrow^{n} b$ for some $n \in Z^{+}$.

(5) - is defined as $a-b$ iff $a \rightarrow \rightarrow a$; let -0 denote - , i.e., -0 $=$ - .

Received by the editors May 9, 1972

AMS (MOS) subject classifications (1970). Primary $20 \mathrm{M10}$.

Key words and phrases. Semigroups, semilattice decomposition, minimal sequence, rank.

(l) The author is supported by a National Science Foundation Graduate Fellowship. 
(6) $a-{ }^{n+1} b$ iff there exists $x \in S$ such that $a-n x-b$.

(7) $a-{ }^{\infty} b$ iff $a-{ }^{n} b$ for some $n \in Z^{+} .-^{\infty}$ is an equivalence relation.

The following theorem and corollary are due to Tamura [8] or [9].

Theorem [Tamura]. Let $S$ be a semigroup. Then $\rightarrow^{\infty} \cap\left(\rightarrow^{\infty}\right)^{-1}$ is the finest semilattice congruence on $S$ and each component is S-indecomposable.

Corollary [Tamura]. Let $S$ be an S-indecomposable semigroup. Then $\rightarrow^{\infty}$ is the universal congruence on $S$.

We generalize these results to:

Theorem 1.1. Let $S$ be a semigroup. Then $-\infty$ is the finest semilattice congruence on $S .-^{\infty}$ is also the equivalence relation generated by the relations $a b \equiv a b a \equiv b a$, for all $a, b \in S^{1}$ and $a b \in S$.

Corollary 1.2. Let $S$ be an S-indecomposable semigroup. Then $-\infty$ is the universal congruence on $S$.

It is easy to deduce Tamura's result from ours.

To prove Theorem 1.1, we need the following

Lemma 1.3. Let $\sigma$ be an equivalence relation on a semigroup $S$ satisfying $x y \sigma x y x \sigma y x$ for all $x, y \in S^{1}$. Then for all $a, b, c, d \in S^{1}$ (with the convention 1 o 1),

(1) $a b c \sigma a b^{i} c$ for all $i \in Z^{+}$,

(2) $a b c d \sigma a c b d$,

(3) $a-{ }^{\infty} b$ implies xay $\sigma$ xby for all $x, y \in S^{1}$.

In partiular ${ }^{\infty} \subseteq \sigma$.

Proof. (1) $a b c \sigma c a b \sigma b(c a) b=(b c)(a b) \sigma(a b)(b c)=a b^{2} c . \quad a b^{i} c=$ $\left(a b^{i-1}\right) b c \sigma\left(a b^{i-1}\right) b^{2} c=a b^{i+1} c$.

(2) Using (1), for any $A, B, C \in S^{\prime}$,

$$
\begin{aligned}
& A B C \sigma A(B C)(B C) \sigma(A B C B C)(A B C B C) \\
&=(A B)(C B C A)(B C)^{2} \sigma(A B)(C B C A) B C \\
& \sigma(A B)(C B C A)(C B C A) B C \\
&= A(B C)^{2}(A C B C A B C) \sigma A(B C)(A C B C A B C) \\
&=(A B C A C B)(C A B C) \sigma(A B C A C B)(A B C A C B)(C A B C) \\
&=(A B C A C B A)(B C)(A C B)(C A B C) \\
& \sigma(A B C A C B A)(B C)(B C)(A C B)(C A B C) \\
&=(A B C A C B A B)(C B C A)^{2} B C \sigma(A B C A C B A B)(C B C A) B C \\
&=(A B C A C B A)(B C)^{2}(A B C) \sigma(A B C A C B A)(B C)(A B C) \\
&=(A B C)(A C B)(A B C)^{2} \sigma(A B C)(A C B)(A B C) .
\end{aligned}
$$


In short $A B C \sigma(A B C)(A C B)(A B C)$. Interchanging $B$ and $C$, we have $A C B$ o $(A C B)(A B C)(A C B)$. But $(A B C)(A C B)(A B C)$ o $(A B C)(A C B)$ o $(A C B)(A B C)(A C B)$. So $A B C \sigma A C B$.

Thus $a b c d \sigma d(a b c)=(d a) b c \sigma(d a) c b=d(a c b) \sigma(a c b) d$.

(3) First suppose $a-b$. So we solve sat $=b^{i}, s^{\prime} b t^{\prime}=a^{j}$. Then using (1), (2), we have $x a b y \sigma x a b^{i} y=$ xasaty $\sigma$ xsaaty $\sigma$ xsaty $=x b^{i} y \sigma x b y$. Similarly, $x$ bay $\sigma$ xay. So by (2), xay $\sigma$ xby. Now assume $a-{ }^{n} b, n \geq 1$. So $a-a_{1}-\cdots$ $-a_{n}-b$. By the above $x a y \sigma x a_{1} y, \quad x a_{i} y \sigma x a_{i+1} y(i=1, \ldots, n-1)$, $x a_{n} y \sigma x b y$. Thus, xay $\sigma$ xby.

Thus $a-{ }^{\infty} b$ implies $x a y \sigma x b y$ for all $x, y \in S^{1}$.

Proof of Theorem 1.1. Consider the following.

$$
x y \equiv x y x \equiv y x, \text { for all } x, y \in S^{1}, x y \in S .
$$

Let $a, b \in S^{1}$. Then $a b a\left|(a b)^{2}, a b\right| a b a$. So $a b a-a b$. Now $a b\left|(b a)^{2}, b a\right|$ $(a b)^{2}$. Thus $a b-b a$. So $a b-\infty a b a-\infty b a$. Thus $-\infty$ is an equivalence relation satisfying (*). By Lemma 1.3 , we conclude that $-\infty$ is the smallest equivalence relation satisfying (*). In the same lemma, replacing $\sigma$ by $-\infty$, we have $-\infty$ is a semilattice congruence. Since any semilattice congruence satisfies (*), we have that $-\infty$ is the finest semilattice congruence on $S$.

Corollary 1.2 is now immediate. We will need the following lemmas later.

Lemma 1.4. Let $S$ be a semilattice of semigroups $S_{\alpha}(\alpha \in \Omega), \delta$ the corresponding semilattice congruence.

(1) Let $\alpha \in \Omega$, with $a, b \in S_{\alpha}$. If $a \rightarrow b$ in $S$, then $a \rightarrow b$ in $S_{\alpha}$.

(2) Let $a \in S_{\alpha}, b \in S_{\beta}, a \rightarrow b$. Then $\alpha \geq \beta$.

(3) Let $a, b \in S$ with $a-b$. Then for some $\alpha \in \Omega, a, b \in S_{\alpha}$ and $a-b$ in $S_{\alpha}$.

Proof. (1) For some $x, y \in S^{1}, x a y=b^{i}$. So $b^{i} x a y b^{i}=b^{3 i}$. Then $b^{i} x$ $=\operatorname{xayx} \delta x a y=b^{i} \delta b$. So $b^{i} x \in S_{\alpha}$. Similarly $y b^{i} \in S_{\alpha}$. So $a \mid b^{3 i}$ in $S_{\alpha}$, whence $a \rightarrow b$ in $S_{\alpha}$.

(2) $\alpha \rightarrow \beta$ in $\Omega$. Since $\Omega$ is a semilattice we deduce $\alpha \mid \beta$ in $\Omega$ and then that $\alpha \geq \beta$.

(3) Let $a \in S_{\alpha}, b \in S_{\beta}$. By (2), $\alpha \geq \beta, \beta \geq \alpha$ and so $\alpha=\beta$. By (1), $a-b$ in $S_{\alpha \cdot}$

Lemma 1.5. Let $S$ be a semigroup and $a, b, c \in S$.

(1) Let $i \in Z^{+}$. Then $a \rightarrow b^{i}$ implies $a \rightarrow b$.

(2) $a \mid b \rightarrow c$ implies $a \rightarrow c$.

(3) Let $i, j \in Z^{+}$. Then $a^{i}-b^{j}$ implies $a-b$.

Lemma 1.6. (1) Let $S$ be a semigroup with an ideal I and $a, b \in S$. Suppose $b$ is not nilpotent in $S / I$, and $a \rightarrow b$ in $S / I$. Then $a \rightarrow b$ in $S$.

(2) Let $S$ be a semigroup with zero, and suppose $a \in S$. Then $0 \rightarrow a$ if and only if $a$ is nilpotent. 
Proof. (1) We can solve $x a y=b^{i}$ in the semigroup $S / I$. Since $b$ is not nilpotent in $S / I, b^{i} \in S \backslash I$. So $x, a, x a, y, x a y \in S \backslash I$. Thus $x a y=b^{i}$ in $S$.

(2) If $0 \rightarrow a$, then $0 \mid a^{i}$ for some $i \in Z^{+}$. Hence $a^{i}=0$. Conversely if $a^{i}=0$ for some $i \in Z^{+}$, then $0 \mid 0=a^{i}$ whence $0 \rightarrow a$.

2. Minimal sequences.

Definition. Let $S$ be a semigroup, $a, b \in S$.

(1) By a sequence between $a$ and $b$, we mean a (possibly empty) finite sequence $\left\langle x_{i}\right\rangle_{i=1}^{n}$ in $S$ such that $a-x_{1}, x_{i}-x_{i+1}(i=1, \ldots, n-1), x_{n}-b$. We call $n$ the length of $\left\langle x_{i}\right\rangle$. By $n=0$, or $\left\langle x_{i}\right\rangle_{i=1}^{n}$ empty, we mean $a-b$. We say $\left\langle x_{i}\right\rangle$ is minimal if it is nonempty and there is no sequence of smaller length (including the empty sequence) between $a$ and $b$.

(2) By a sequence from $a$ to $b$, we mean a (possibly empty) finite sequence $\left\langle x_{i}\right\rangle_{i=1}^{n}$, such that $a \rightarrow x_{1}, x_{i} \rightarrow x_{i+1}(i=1, \ldots, n-1), x_{n} \rightarrow b$. Again $n$ is the length of the sequence, and by $n=0$ (or $\left\langle x_{i}\right\rangle$ empty) we mean $a \rightarrow b$. $\left\langle x_{i}\right\rangle$ is minimal if it is nonempty and there is no sequence of smaller length (including the empty sequence) from $a$ to $b$.

Lemma 2.1. Let $S$ be a semigroup with $a, b \in S$.

(1) Let $\left\langle x_{i}\right\rangle_{i=1}^{n},\left\langle y_{i}\right\rangle_{i=1}^{n}$ be two sequences between $a$ and $b$ of the same length. If $\left\langle x_{i}\right\rangle$ is minimal, then so is $\left\langle y_{i}\right\rangle$.

(2) Let $S$ be $\delta$-indecomposable. Then either $a-b$ or there is a minimal sequence between $a$ and $b$.

(3) Let $S$ be S-indecomposable. Then either $a \rightarrow b$ or there is a minimal sequence from $a$ to $b$.

Proof. (1) Obvious.

(2) and (3) are trivial using Corollary 1.2.

Lemma 2.2. Let $S$ be a semilattice of semigroups $S_{\alpha}(\alpha \in \Omega)$.

(1) Let $a, b \in S$, with $a$ sequence $\left\langle x_{i}\right\rangle_{i=1}^{n}$ between $a$ and $b$. Then $a, b$ and all the $x_{i}$ 's lie in some $S_{\alpha}$. Moreover $\left\langle x_{i}\right\rangle$ is a sequence between $a$ and $b$ in $S_{\alpha \cdot}$.The minimal sequences between $a$ and $b$ in $S$ are exactly those in $S_{\alpha}$.

(2) Let $\alpha \in \Omega$, with $a, b \in S_{\alpha}$. Let $\left\langle x_{i}\right\rangle_{i=1}^{n}$ be a sequence from $a$ to $b$ in $S$. Then all the $x_{i}$ 's lie in $S_{\alpha}$ and $\left\langle x_{i}\right\rangle$ is a sequence from $a$ to $b$ in $S_{\alpha}$. The minimal sequences from $a$ to $b$ in $S$ are exactly those in $S_{a}$.

(3) Let $a \in S_{\alpha}, b \in S_{\beta}$. Suppose there exists a sequence from $a$ to $b$ in $S$. Then $\alpha \geq \beta$.

Proof. (1) That $x_{i}$ 's, $a, b$ lie in some $S_{a}$ and that $\left\langle x_{i}\right\rangle$ is a sequence between $a$ and $b$ within $S_{a}$ follow from Lemma 1.4. So a minimal sequence between $a$ and $b$ in $S$ is a sequence between $a$ and $b$ in $S_{\alpha}$ and obviously minimal in $S_{\alpha}$. Let $\left\langle y_{i}\right\rangle$ be a minimal sequence between $a$ and $b$ in $S_{\alpha}$. Let $\left\langle z_{i}\right\rangle$ be a sequence between $a$ and $b$ in $S$. By the above, $\left\langle z_{i}\right\rangle$ is a sequence between $a$ and $b$ in $S_{\alpha}$. So $\left\langle z_{i}\right\rangle$ has length at least that of $\left\langle y_{i}\right\rangle$. So $\left\langle y_{i}\right\rangle$ is minimal in $S$. 
(2) If $\left\langle x_{i}\right\rangle$ is empty, $a \rightarrow b$ in $S$ and so in $S_{a}$, by Lemma 1.4. Otherwise let $x_{i} \in S_{\alpha, p} i=1, \ldots, n$. Then $a \rightarrow x_{1} \rightarrow \cdots \rightarrow x_{n} \rightarrow b$. By Lemma 1.4, $\alpha \geq \alpha_{1}$ $\geq \cdots \geq \alpha_{n} \geq \alpha$. Consequently, $\alpha=\alpha_{1}=\cdots=\alpha_{n}$. Now $\left\langle x_{i}\right\rangle$ is a sequence from $a$ to $b$ within $S_{\alpha}$, by Lemma 1.4. The rest follows as in (1).

(3) If the sequence is empty, $a \rightarrow b$ and so by Lemma 1.4, $\alpha \geq \beta$. Otherwise, $a \rightarrow x_{1} \cdots x_{n} \rightarrow b, x_{i} \in S_{\alpha_{i}}$. By Lemma 1.4, $\alpha \geq \alpha_{1} \geq \cdots \geq \alpha_{n} \geq \beta$. So $\alpha$ $\geq \beta$.

Definition. (1) A semigroup $S$ is a $\Gamma$-semigroup iff for any $a, b \in S$, either $a \rightarrow b$ or $b \rightarrow a$. Clearly any semigroup $S$ with J-classes linearly ordered (equivalently the ideals are linearly ordered or still equivalently for any $a, b \in S$, $a \mid b$ or $b \mid a)$ is a $\Gamma$-semigroup. Such an example is the full transformation semigroup. The null semigroup with more than one element is a $\Gamma$-semigroup, but its 2 -classes are not linearly ordered.

(2) $S$ is a $\Gamma^{*}$-semigroup iff $S$ is a semilattice of $\Gamma$-semigroups.

Lemma 2.3. Let $S$ be a semigroup. Then the following are equivalent.

(1) $S$ is a $\Gamma^{*}$-semigroup.

(2) $S$ is a semilattice of $\Gamma^{*}$-semigroups.

(3) The S-indecomposable components of $S$ are $\Gamma$-semigroups.

Proof. (1) $\Rightarrow$ (2). Obvious.

(2) $\Rightarrow$ (3). Let $S$ be a semilattice of $\Gamma^{*}$-semigroups $S_{\alpha}(\alpha \in \Omega)$. Let $T$ be an $\delta$ indecomposable component of $S$. Then $T \subseteq S_{\alpha}$ for some $\alpha \in \Omega$. $S_{\alpha}$ is a semilattice of $\Gamma$-semigroups $U_{\beta}$. So $T \subseteq U_{\beta}$ for some $\beta$. Let $a, b \in T$. Then $a, b \in U_{\beta}$. So $a \rightarrow b$ or $b \rightarrow a$ in $U_{\beta}$ and hence in $S$. By Lemma 1.4, $a \rightarrow b$ or $b \rightarrow a$ in $T$. Consequently $T$ is a $\Gamma$-semigroup.

(3) $\Rightarrow(1)$. Obvious.

Definition. Let $a, b \in S$. Then $a \leadsto b$ iff $a^{i} \rightarrow b$ for all $i \in Z^{+}$.

Lemma 2.4. Let $S$ be a semigroup with $a, b, c \in S$.

(1) If $a \rightarrow b \leadsto c$, then $a \rightarrow c$.

(2) If $S$ is $a \Gamma$-semigroup, then either $a \leadsto b$ or $b \leadsto a$.

Proof. (1) $a \mid b^{i} \rightarrow c$ for some $i \in Z^{+}$. So $a \rightarrow c$.

(2) Suppose $a \nrightarrow b$. Then $a^{i} \nrightarrow b$ for some $i \in Z^{+}$. So for any $k \in Z^{+}$, $a^{i} \nrightarrow b^{k}$. Hence $b^{k} \rightarrow a^{i}$ and so $b^{k} \rightarrow a$. Since $k$ is arbitrary, $b \leftrightarrow a$.

Theorem 2.5. Let $S$ be a $\Gamma^{*}$-semigroup with $a, b \in S$. Let $\left\langle x_{i}\right\rangle_{i=1}^{n},\left\langle y_{i}\right\rangle_{i=1}^{n}$ be two minimal sequences between $a$ and $b$. Then $x_{i}-y_{i}$ for $i=1, \ldots, n$. We can further conclude (if $n>1)$ that for $i=1, \ldots, n-1$, either $x_{i}-y_{i+1}$ or $y_{i}-x_{i+1}$.

Proof. $S$ is a semilattice of $\Gamma$-semigroups $S_{\alpha}(\alpha \in \Omega)$. Using Lemma 2.2, we deduce that if the theorem is true for each $S_{\alpha}$, it is true for $S$. So we can assume that $S$ is a $\Gamma$-semigroup. We use Lemma 2.4 without further remark. 
First we prove the theorem for $n=1$. We have

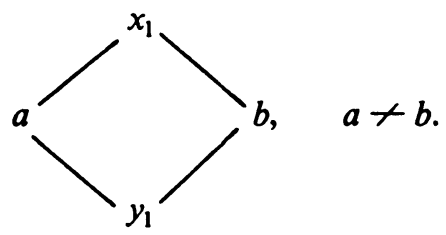

Now $a \leadsto b$ or $b \leadsto a$. By symmetry, we assume $a \leadsto b$. Since $a \neq b$, we conclude that $b \nrightarrow a$. Now either $x_{1} \leadsto a$ or $a \leadsto x_{1}$. If $x_{1} \leadsto a$, we have (since $b \rightarrow x_{1}$ ) that $b \rightarrow a$, a contradiction. So, $a \leadsto x_{1}$. Since $y_{1} \rightarrow a$, we have $y_{1} \rightarrow x_{1}$. Similarly $x_{1} \rightarrow y_{1}$. Thus $x_{1}-y_{1}$.

We now proceed by induction on $n$. We have,

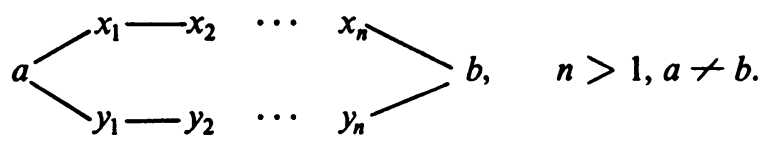

Now either $a \leadsto b$ or $b \leadsto a$. By symmetry, we assume $a \leadsto b$. Since $a \not b$, $b \nrightarrow a$. Again, either $x_{n} \leadsto a$ or $a \leadsto x_{n}$. If $x_{n} \leadsto a$, we obtain (since $b \rightarrow x_{n}$ ), $b \rightarrow a$, a contradiction. So $a \leadsto x_{n}$. Now assume $a \leadsto x_{j+1}, j \geq 1$. Then $x_{j+1} \nrightarrow a$, for otherwise, $a-x_{j+1}$ and so $\left\langle x_{i}\right\rangle_{i=j+1}^{n}$ is a sequence between $a$ and $b$, contradicting the minimality of $\left\langle x_{i}\right\rangle_{i=1}^{n}$. Now either $x_{j} \leadsto a$ or $a \leadsto x_{j}$. If $x_{j} \leadsto a$, then since $x_{j+1} \rightarrow x_{j}$ we have $x_{j+1} \rightarrow a$, a contradiction. So $a \leadsto x_{j}$. Thus $a \leadsto x_{i}$ for all $i=1, \ldots, n$. Similarly $a \leadsto y_{i}$ for all $i=1, \ldots, n$. In particular $a \leadsto x_{1}$, $a \leadsto y_{1}$. Since $x_{1} \rightarrow a$, we have $x_{1} \rightarrow y_{1}$. Similarly, $y_{1} \rightarrow x_{1}$. Thus $x_{1}-y_{1}$. We further have $y_{1} \rightarrow a \leadsto x_{2}$, and so $y_{1} \rightarrow x_{2}$. Similarly, $x_{1} \rightarrow y_{2}$. Now $x_{1} \leadsto y_{1}$ or $y_{1} \leadsto x_{1}$. We assume $x_{1} \leadsto y_{1}$, the other case being taken care of similarly. So $x_{2} \rightarrow x_{1} \leadsto y_{1}$ and hence $x_{2} \rightarrow y_{1}$. Since we already established $y_{1} \rightarrow x_{2}$, we have $x_{2}-y_{1}$. Thus we obtain:
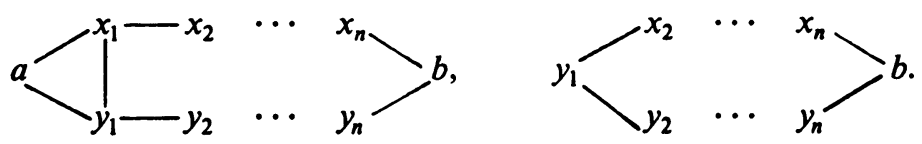

In the figure on the right, the sequence $\left\langle y_{i}\right\rangle_{i=2}^{n}$ is a minimal sequence between $y_{1}$ and $b$. This is because a sequence between $y_{1}$ and $b$ of length less than $n-1$ would produce a sequence between $a$ and $b$ of length less than $n$, contradicting the minimality of $\left\langle y_{i}\right\rangle_{i=1}^{n}$. By Lemma $2.1,\left\langle x_{i}\right\rangle_{i=2}^{n}$ is a minimal sequence between $y_{1}$ and $b$. By our induction hypothesis, we have $x_{i}-y_{i}$, for $i \geq 2$. Also if $n>2$, $x_{i}-y_{i+1}$, or $y_{i}-x_{i+1}, i=2, \ldots, n-1$. Since we already know $x_{1}-y_{1}$, $y_{1}-x_{2}$, the theorem is proved.

We will see later that Theorem 2.5 is not true for arbitrary semigroups, even for $n=1$.

Problem 2.6. In Theorem 2.5 can we conclude that $x_{i}-y_{i+1}$ and $y_{i}-x_{i+1}$, $i=1, \ldots, n-1$ ? 
Problem 2.7. Call a sequence $\left\langle x_{i}\right\rangle$ between $a$ and $b$ indecomposable if $\left\langle x_{i}\right\rangle$ is nonempty and no proper subsequence of $\left\langle x_{i}\right\rangle$ is a sequence between $a$ and $b$. Clearly a minimal sequence is indecomposable but not conversely. An indecomposable sequence of length 1 is minimal. Is Theorem 2.5 true for indecomposable sequences of the same length? For $n \leq 2$, the proof goes through.

Lemma 2.8. Let $S$ be $a \Gamma$-semigroup and $a, b \in S$. Let $\left\langle x_{i}\right\rangle_{i=1}^{n}$ be a minimal sequence from $a$ to $b$. Then $\left\langle x_{i}\right\rangle_{i=1}^{n}$ is a minimal sequence between $a$ and $b$.

Proof. We have $a \rightarrow x_{1} \cdots x_{n} \rightarrow b, n \geq 1$. Set $x_{0}=a, x_{n+1}=b$. For $n \geq i$ $\geq 1, x_{i} \leadsto x_{i+1}$ implies $x_{i-1} \rightarrow x_{i+1}$ (since $x_{i-1} \rightarrow x_{i}$ ) contradicting the minimality of $\left\langle x_{i}\right\rangle_{i=1}^{n}$. So $x_{i+1} \leadsto x_{i}$. Thus $x_{i}-x_{i+1}, n \geq i \geq 1$. Now $a \nrightarrow x_{2}$ by minimality of $\left\langle x_{i}\right\rangle_{i=1}^{n}$. So $x_{2} \leadsto a$. Since $x_{1} \rightarrow x_{2}$, we have $x_{1} \rightarrow a$. So $a-x_{1}$. Consequently, $\left\langle x_{i}\right\rangle_{i=1}^{n}$ is a sequence between $a$ and $b$. Since any sequence between $a$ and $b$ is a sequence from $a$ to $b$, we have that $\left\langle x_{i}\right\rangle$ is a minimal sequence between $a$ and $b$.

Corollary 2.9. Let $S$ be a $\Gamma$-semigroup with $a, b, c \in S$. Let $\left\langle x_{i}\right\rangle_{i=1}^{n}$ and $\left\langle y_{i}\right\rangle_{i=1}^{n}$ be minimal sequences of the same length from $b$ to $a$ and $c$ to $a$ respectively. Then $x_{i}-y_{i}$ for $i=1, \ldots, n$. For $n>1$, we can further conclude that for each $i=1, \ldots, n-1$ either $x_{i}-y_{i+1}$ or $y_{i}-x_{i+1}$.

Proof. Now either $x_{1} \leadsto y_{1}$ or $y_{1} \leadsto x_{1}$. By symmetry, we assume $x_{1} \leadsto y_{1}$. Since $b \rightarrow x_{1}$, we have $b \rightarrow y_{1}$. Thus $\left\langle y_{i}\right\rangle_{i=1}^{n}$ is a sequence from $b$ to $a$. Since $\left\langle x_{i}\right\rangle_{i=1}^{n}$ is minimal, we obtain that $\left\langle y_{i}\right\rangle_{i=1}^{n}$ is also a minimal sequence from $b$ to $a$. By Lemma 2.8, $\left\langle x_{i}\right\rangle$ and $\left\langle y_{i}\right\rangle$ are minimal sequences between $b$ and $a$. By Theorem 2.5 , we are done.

Problem 2.10. Is Corollary 2.9 true for $\Gamma^{*}$-semigroups?

\section{Rank and semirank.}

Definition. Let $S$ be a semigroup.

(1) The rank $\rho_{1}(S)$ of a semigroup $S$ is zero if there is no minimal sequence between any two points. Otherwise $\rho_{1}(S)$ is the supremum of the lengths of the minimal sequences between points in $S$.

(2) The semirank $\rho_{2}(S)$ of $S$ is zero if there is no minimal sequence from a point to another in $S$. Otherwise $\rho_{2}(S)$ is the supremum of the lengths of the minimal sequences from one point to another in $S$.

The following is an easy consequence of Lemma 2.1.

Lemma 3.1. Let $S$ be an $\mathcal{S}$-indecomposable semigroup, and $a, b \in S$. Then there exists $a$ sequence between $a$ and $b$ of length at most $\rho_{1}(S) .(2)$ Also there exists $a$ sequence from $a$ to $b$ of length at most $\rho_{2}(S)$.

Lemma 3.2. Let $S$ be the semilattice of $\delta$-indecomposable semigroups $S_{\alpha}(\alpha$ $\in \Omega)$. Then

(1) $\rho_{1}(S)=\sup _{\alpha \in \Omega} \rho_{1}\left(S_{\alpha}\right)$,

(2) $\rho_{2}(S)=\sup _{\alpha \in \Omega} \rho_{2}\left(S_{\alpha}\right)$.

(2) If $\rho_{1}(S)=\infty$, the length is less than $\rho_{1}(S)$. Similarly for $\rho_{2}(S)$. 
Proof. (1) Immediate from Lemma 2.2.

(2) By Lemma 2.2, we have $\rho_{2}\left(S_{\alpha}\right) \leq \rho_{2}(S)$ for all $\alpha \in \Omega$. So $\sup _{\alpha \in \Omega} \rho_{2}\left(S_{\alpha}\right)$ $\leq \rho_{2}(S)$. Now let $a, b \in S,\left\langle x_{i}\right\rangle_{i=1}^{n}$ a minimal sequence from $a$ to $b$. We have to show that $n \leq \sup _{\alpha \in \Omega} \rho_{2}\left(S_{\alpha}\right)$. Let $a \in S_{\gamma}, b \in S_{\beta}$. By Lemma 2.2, $\gamma \geq \beta$. So $a b \in S_{\beta}$. By Lemma 3.1, there exists a sequence $\left\langle y_{i}\right\rangle_{i=1}^{k}$ from $a b$ to $b, k$ $\leq \rho_{2}\left(S_{\beta}\right)$. Since $a \mid a b$, by Lemma $1.5,\left\langle y_{i}\right\rangle_{i=1}^{k}$ is a sequence from $a$ to $b$. By minimality of $\left\langle x_{i}\right\rangle_{i=1}^{n}$ we have $n \leq k \leq \rho_{2}\left(S_{\beta}\right) \leq \sup _{\alpha \in \Omega} \rho_{2}\left(S_{\alpha}\right)$. Thus $\rho_{2}(S)$ $\leq \sup _{\alpha \in \Omega} \rho_{2}\left(S_{\alpha}\right)$. Combined with the previous result, $\rho_{2}(S)=\sup _{\alpha \in \Omega} \rho_{2}\left(S_{\alpha}\right)$.

A semigroup $S$ is archimedean if and only if for all $a, b \in S, a \rightarrow b$ (see [3], [7] and [8]).

Theorem 3.3. Let $S$ be a semigroup.

(1) $\rho_{1}(S)$ is the smallest $n \leq \infty$ for which $-n$ is transitive (i.e., $-^{n}=-\infty$ or equivalently $-^{n}=-^{n+1}$ ).

(2) $\rho_{2}(S)$ is the smallest $n \leq \infty$ for which $\rightarrow^{n}$ is transitive (i.e., $\rightarrow^{n}=\rightarrow^{\infty}$ or equivalently $\rightarrow^{n}=\rightarrow^{n+1}$ ).

(3) Let $S$ be a semilattice of semigroups $S_{\alpha}(\alpha \in \Omega)$. Then $\rho_{i}(S)$ $=\sup _{\alpha \in \Omega} \rho_{i}\left(S_{\alpha}\right), i=1,2$.

(4) $\rho_{2}(S) \leq \rho_{1}(S)$.

(5) $\rho_{1}(S)=0$ if and only if $\rho_{2}(S)=0$ if and only if $S$ is a semilattice of archimedean semigroups.

(6) If $S$ is a $\Gamma^{*}$-semigroup, $\rho_{1}(S)=\rho_{2}(S)$.

(7) $A$ finite semigroup has finite semirank and rank.

Proof. (1) and (2) are easy consequences of the definition.

(3) For each $\alpha \in \Omega, S_{\alpha}$ is the semilattice of the $\delta$-indecomposable components of $S$, contained in $S_{\alpha}$. So the $\delta$-indecomposable components of $S$ are just those of all of the $S_{\alpha}$ 's. Now the result follows from Lemma 3.2.

(4) By (3), we can assume $S$ is $\delta$-indecomposable. Let $a, b \in S$ and $\left\langle x_{i}\right\rangle_{i=1}^{n}$ a minimal sequence from $a$ to $b$. By Lemma 3.1, there exists a sequence $\left\langle y_{i}\right\rangle_{i=1}^{m}$ between $a$ and $b$, such that $m \leq \rho_{1}(S)$. But $\left\langle y_{i}\right\rangle$ can be considered a sequence from $a$ to $b$. By the minimality of $\left\langle x_{i}\right\rangle, n \leq m \leq \rho_{1}(S)$. So $\rho_{2}(S) \leq \rho_{1}(S)$.

(5) Again we can assume $S$ is $S$-indecomposable. Clearly if $S$ is archimedean, it has no minimal sequences and so $\rho_{1}(S)=\rho_{2}(S)=0$. If for $i=1$ or 2 , $\rho_{i}(S)=0$, then by Lemma 3.1, $S$ is archimedean.

(6) By Lemma 2.3 and by (3) and (4) above, we can assume that $S$ is an $\delta$ indecomposable $\Gamma$-semigroup and that $\rho_{2}(S) \leq \rho_{1}(S)$. We have to show $\rho_{1}(S)$ $\leq \rho_{2}(S)$. Let $a, b \in S$ and let $\left\langle x_{i}\right\rangle_{i=1}^{n}$ be a minimal sequence between $a$ and $b$. Then $a \nrightarrow b$. So either $a \nrightarrow b$ or $b \nrightarrow a$. By symmetry we assume $a \nrightarrow b$. By Lemma 2.1, there exists a minimal sequence $\left\langle y_{i}\right\rangle_{i=1}^{m}$ from $a$ to $b$. So $m \leq \rho_{2}(S)$. By Lemma 2.8, $\left\langle y_{i}\right\rangle_{i=1}^{m}$ is a minimal sequence between $a$ and $b$. Thus $n=m$ $\leq \rho_{2}(S)$. So $\rho_{1}(S) \leq \rho_{2}(S)$, whence $\rho_{1}(S)=\rho_{2}(S)$.

(7) Obvious. 
Lemma 3.4. Let $S$ be an S-indecomposable semigroup and $T$ a homomorphic image of $S$. Then $\rho_{i}(T) \leq \rho_{i}(S), i=1,2$.

Proof. Let $a, b \in S, \varphi: S \rightarrow T$ an onto homomorphism. Let there be a minimal sequence $\left\langle y_{i}\right\rangle_{i=1}^{n}$ in $T$, between $\varphi(a)$ and $\varphi(b)$. So $\varphi(a)+\varphi(b)$ and so $a \neq b$. By Lemma 2.1, there exists a minimal sequence $\left\langle x_{i}\right\rangle_{i=1}^{m}$ between $a$ and $b$. Thus $m \leq \rho_{1}(S)$. $\left\langle\varphi\left(x_{i}\right)\right\rangle_{i=1}^{m}$ is a sequence between $\varphi(a)$ and $\varphi(b)$. By minimality of $\left\langle y_{i}\right\rangle_{i=1}^{n}$, we have $n \leq m \leq \rho_{1}(S)$. Thus $\rho_{1}(T) \leq \rho_{1}(S)$. A similar argument shows that $\rho_{2}(T) \leq \rho_{2}(S)$.

Problem 3.5. Is Lemma 3.4 true for arbitrary semigroups?

Theorem 3.6. Let $S$ be an $\delta$-indecomposable semigroup with an ideal I. Then

$$
\rho_{2}(S / I) \leq \rho_{2}(S) \leq \rho_{2}(I)+\rho_{2}(S / I)
$$

Proof. That $\rho_{2}(S / I) \leq \rho_{2}(S)$ follows from Lemma 3.4. By [5] (or [10]), both $I$ and $S / I$ are $\delta$-indecomposable. Let $a, b \in S$. We have to show the existence of a sequence from $a$ to $b$ of length at most $\rho_{2}(I)+\rho_{2}(S / I)$. (3)

Case 1. $a \in S, b \in I$. Then $a b \in I$. So by Lemma 3.1, there exists a sequence $\left\langle y_{i}\right\rangle_{i=1}^{m}$ from $a b$ to $b$ in $I$ such that $m \leq \rho_{2}(I)$. By Lemma $1.5,\left\langle y_{i}\right\rangle_{i=1}^{m}$ is a sequence from $a$ to $b$.

Case 2. $a \in I, b \in S \backslash I, b$ is nilpotent in $S / I$. Then $b^{k} \in I$ for some $k \in Z^{+}$. Then by Lemma 3.1, there exists a sequence $\left\langle y_{i}\right\rangle_{i=1}^{m}$ from $a$ to $b^{k}, m \leq \rho_{2}(I)$. By Lemma $1.5,\left\langle y_{i}\right\rangle_{i=1}^{m}$ is a sequence from $a$ to $b$.

Case 3. $a \in I, b \in S \backslash I, b$ is not nilpotent in $S / I$. So in $S / I, 0 \nrightarrow b$. By Lemma 2.1, there exists a minimal sequence $\left\langle y_{i}\right\rangle_{i=1}^{n}$ from 0 to $b$. So $n \leq \rho_{2}(S / I)$. So in $S / I, 0 \rightarrow y_{1} \rightarrow \cdots \rightarrow y_{n} \rightarrow b$. If $y_{j}$ is nilpotent in $S / I$, for some $j>1$, we would have, by Lemma 1.6, $0 \rightarrow y_{j} \rightarrow \cdots \rightarrow y_{n} \rightarrow b$ contradicting the minimality of $\left\langle y_{i}\right\rangle_{i=1}^{n}$. So $y_{j}$ is not nilpotent for $\left.j\right\rangle 1$. By Lemma $1.6, y_{1} \rightarrow \cdots \rightarrow y_{n} \rightarrow b$ in $S$. Now since $0 \rightarrow y_{1}, y_{1}$ is nilpotent in $S / I$. So by Case 2 , there exists a sequence $\left\langle x_{i}\right\rangle_{i=1}^{m}$ from $a$ to $y_{1}$ in $S$ such that $m \leq \rho_{2}(I)$. So in $S$,

$$
a \rightarrow x_{1} \rightarrow \cdots \rightarrow x_{m} \rightarrow y_{1} \rightarrow \cdots \rightarrow y_{n} \rightarrow b, \quad m+n \leq \rho_{2}(I)+\rho_{2}(S / I) .
$$

Case 4. $a \in S \backslash I, b \in S \backslash I$. If $b$ is nilpotent in $S / I$, then $b^{k} \in I$ for some $k \in Z^{+}$. By Case 1, there exists in $S$ a sequence $\left\langle y_{i}\right\rangle$ from $a$ to $b^{k}$ of length $\leq \rho_{2}(I)$. By Lemma $1.5,\left\langle y_{i}\right\rangle$ is a sequence from $a$ to $b$ in $S$.

Thus we may assume $b$ is not nilpotent in $S / I$. So if $a \rightarrow b$ in $S / I$, then by Lemma 1.6, $a \rightarrow b$ in $S$ and we would be done. So we assume $a \nrightarrow b$ in $S / I$. By Lemma 2.1, there exists a minimal sequence $\left\langle x_{i}\right\rangle_{i=1}^{n}$ in $S / I$ from $a$ to $b$. So $n \leq \rho_{2}(S / I)$. If none of the $x_{i}$ 's is nilpotent, then $\left\langle x_{i}\right\rangle_{i=1}^{n}$ is a sequence from $a$ to $b$ in $S$, by Lemma 1.6. So let some $x_{j}$ be nilpotent in $S / I$. Then $a \rightarrow x_{j} \rightarrow$

(3) The theorem is trivial if $p_{2}(I)=\infty$ or $p_{2}(S / I)=\infty$. So we assume $p_{2}(I)<\infty$ and $\rho_{2}(S / I)<\infty$. 
$\cdots \rightarrow x_{n} \rightarrow b$ in $S / I$. By minimality of $\left\langle x_{i}\right\rangle_{i=1}^{n}, j=1$. Thus $x_{1}$ is nilpotent and $x_{j}$ is not nilpotent for $j>1$. Thus by Lemma $1.6, x_{1} \rightarrow \cdots \rightarrow x_{n} \rightarrow b$ in $S$. From what we proved above there exists a sequence $\left\langle y_{i}\right\rangle_{i=1}^{m}$ in $S$ from $a$ to $x_{1}$ such that $m \leq \rho_{2}(I)$. Thus in $S$,

$$
a \rightarrow y_{1} \rightarrow \cdots \rightarrow y_{m} \rightarrow x_{1} \rightarrow \cdots \rightarrow x_{n} \rightarrow b, \quad m+n \leq \rho_{2}(I)+\rho_{2}(S / I) .
$$

Problem 3.7. Is Theorem 3.6 true for arbitrary semigroups? In Theorem 3.6, can we replace $\rho_{2}$ by $\rho_{1}$ ?

Consider the following condition on semigroups:

$a \in S$ implies there exists a fixed $n=n(a) \in Z^{+}$ such that for all $i \in Z^{+}, a^{i n} \mid a^{n}$.

Clearly any semigroup with a power of each element lying in a subgroup (in particular a periodic semigroup) satisfies (A).

Lemma 3.8. Let $S$ be a semigroup satisfying (A). Let $a, b \in S, k \in Z^{+}$, such that $b \rightarrow a^{k}$. Then $b \mid a^{n(a)}$.

Proof. For some $i \in Z^{+}, b\left|a^{i}\right| a^{i n(a)} \mid a^{n(a)}$. So $b \mid a^{n(a)}$.

Theorem 3.9. Let $S$ be a semigroup satisfying (A). Suppose $\rho_{2}(S) \leq 1$. Then $\rho_{1}(S) \leq 4$.

Proof. Let $\delta$ be the finest semilattice congruence on $S$ and $S_{\alpha}(\alpha \in S / \delta)$ the $\delta$ indecomposable components of $S$. Let $a \in S_{\alpha}$. By (A), there exists $n=n(a)$ $\in Z^{+}$such that for all $i \in Z^{+}, a^{(i+2) n} \mid a^{n}$ in $S$. So there exists $x, y \in S^{1}$ such that $x a^{n} a^{i n} a^{n} y=a^{n}$. But then $x a^{n}=x\left(x a^{(i+2) n} y\right) \delta x a^{(i+2) n} y=a^{n} \delta a$. So $x a^{n}$ $\in S_{\alpha}$. Similarly $a^{n} y \in S_{\alpha}$. Thus $a^{i n} \mid a^{n}$ in $S_{\alpha}$. Consequently each $S_{\alpha}$ satisfies (A). By Theorem 3.3, it suffices to prove the theorem for each $S_{\alpha}$. Consequently we may and do assume that $S$ is an $\delta$-indecomposable semigroup. Let $a, b \in S$. We have to show the existence of a sequence between $a$ and $b$ of length at most 4 . We use Lemma 3.1 and Lemma 3.8 without further remark. Let $n_{1}=n(a)$, $n_{2}=n(b)$. There exists $c \in S$ such that $a^{n_{1}} \rightarrow c \rightarrow b^{n_{2}}$. Set $n_{3}=n(c)$. So $a^{n_{1}} \mid c^{n_{3}}$. There exists $d_{1} \in S$ such that $c^{n_{3}} \rightarrow d_{1} \rightarrow a^{n_{1}}$. Set $m_{1}=n\left(d_{1}\right)$. So $d_{1}\left|a^{n_{1}}\right| c^{n_{3}} \mid d_{1}^{m_{1}}$. So $d_{1} \mid c^{n_{3}}$ and $a^{n_{1}} \mid d_{1}^{m_{1}}$. Thus $c^{n_{3}}-d_{1}-a^{n_{1}}$. By Lemma 1.5, $c-d_{1}-a$. Now since $c \rightarrow b^{n_{2}}, c \mid b^{n_{2}}$. There exists $d_{2} \in S$ such that $b^{n_{2}} \rightarrow d_{2} \rightarrow c$. Let $m_{2}$ $=n\left(d_{2}\right)$. Then $c\left|b^{n_{2}}\right| d_{2}^{m_{2}}$. Thus $c \mid d_{2}^{m_{2}}$ and hence, $b^{n_{2}} \rightarrow d_{2}-c$. There exists $d_{3} \in S$ such that $d_{2}^{m_{2}} \rightarrow d_{3} \rightarrow b^{n_{2}}$. Let $m_{3}=n\left(d_{3}\right)$. So $d_{3}\left|b^{n_{2}}\right| d_{2}^{m_{2}} \mid d_{3}^{m_{3}}$. Hence $d_{3} \mid d_{2}^{m_{2}}$ and $b^{n_{2}} \mid d_{3}^{m_{3}}$. Thus $d_{2}^{m_{2}}-d_{3}-b^{n_{2}}$. By Lemma 1.5, $d_{2}-d_{3}-b$. Hence $c-d_{2}-d_{3}-b$. Consequently $a-d_{1}-c-d_{2}-d_{3}-b$, and the theorem is proved.

Problem 3.10. Can the bound on $\rho_{1}$ be improved in Theorem 3.9? Does a semigroup of finite semirank necessarily have finite rank? 
4. Examples. It can be deduced from Corollary 1.2 or from Tamura's corollary that a 0 -simple semigroup is $\delta$-indecomposable if and only if it has a nonzero nilpotent element. Also notice that for an $\delta$-indecomposable semigroup $S$ with zero, $\rho_{1}(S)=0$ if and only if $\rho_{2}(S)=0$ if and only if $S$ is nil.

Example 4.1. Let $S$ be a 0 -simple semigroup with a nonzero nilpotent element $b$. Then $0-b$. If $x$ is a nonnilpotent element in $S$, then $0-b-x, 0 \rightarrow b \rightarrow x$ are minimal sequences. So $\rho_{1}(S)=\rho_{2}(S)=1$.

Example 4.2. Let $S_{1}, S_{2}$ be two 0 -simple semigroups with $b_{1} \in S_{1}, b_{2} \in S_{2}$ being nonzero nilpotent elements of $S_{1}$ and $S_{2}$ respectively. Identify the zeros of $S_{1}$ and $S_{2}$. Let $S=S_{1} \cup S_{2}$ and $S_{1} \cap S_{2}=S_{1} S_{2}=S_{2} S_{1}=\{0\}$. Let $x \in S_{1}$, $y \in S_{2}$ be nonnilpotent. Then $x-b_{1}-b_{2}-y$ is a minimal sequence between $x$ and $y$. So $\rho_{1}(S)=2$. But $x \rightarrow b_{2} \rightarrow y, y \rightarrow b_{1} \rightarrow x$, whence $\rho_{2}(S)=1$. Thus the rank of a semigroup can be strictly larger than the semirank.

Example 4.3. Let $S, S_{1}, S_{2}$ be as in Example 4.2. This time choose $b_{1} \in S_{1}$, $b_{2} \in S_{2}$ such that $b_{1}, b_{2} \neq 0, b_{1}^{2}=b_{2}^{2}=0$. Let $T=S \cup\{u\}, u \notin S$. Define $u^{2}=0, x u=x b_{1}, u x=b_{1} x, u y=b_{2} y, y u=y b_{2}$ where $x \in S_{1}, y \in S_{2}$. It can be seen that $T$ is a semigroup with ideal $S$. If $s_{1} \in S_{1}, s_{2} \in S_{2}$, then $u\left|b_{1}\right| s_{1}$, $u\left|b_{2}\right| s_{2}, s_{1}\left|u^{2}=0, s_{2}\right| u^{2}=0$. Hence $s_{1}-u-s_{2}$. Thus $\rho_{1}(T)=1$. But $\rho_{1}(S)=2$ by Example 4.2. Thus the rank of an ideal of a semigroup can be greater than that of the semigroup. Can a similar thing happen with semirank? Can it happen for $\Gamma$-semigroups? Can the rank of a semigroup be less than that of an ideal by an arbitrary number?

Example 4.4. We are now going to construct $\delta$-indecomposable $\Gamma$-semigroups of every rank (and hence semirank). A group is an example of an $\delta$-indecomposable, $\Gamma$-semigroup of rank and semirank zero. Now let $\left\langle S_{i}\right\rangle_{i \in Z^{+}}$be a sequence of 0 -simple semigroups. Assume $S_{i}$ has zero $0_{i}$, a nonzero nilpotent element $b_{i}$, $b_{i}^{2}=0_{i}$, and a nonzero idempotent $e_{i}$. (For instance $S_{i}$ could be a completely 0 simple semigroup which is not a Clifford semigroup.) Now identify $e_{i}$ and $\mathbf{0}_{i+1}$. Thus $S_{i} \cap S_{i+1}=\left\{e_{i}\right\}=\left\{0_{i+1}\right\}$. Let $S=\cup_{i \in Z^{+}} S_{i}$. Set $I_{i}=\cup_{j \leq i} S_{j}$. We define multiplication on $S$ by defining multiplication on each $I_{i}$. $I_{1}=S_{1}$ is a semigroup. Assume multiplication has been defined on $I_{i}$. Let $x \in I_{i}$ and $y \in S_{i+1}$. Then define $x y=x e_{i}, y x=e_{i} x$. Then it can be seen that the multiplication is consistent with the previous (i.e., when $x$ or $y=e_{i}=0_{i+1}$ ), and also that now $I_{i+1}$ is a semigroup. Consequently, we obtain a semigroup $S$. Let $0_{1}=0$. Then 0 is the zero of $S$, and each $I_{i}$ is an ideal of $S$. Now let $x_{i}$ be a nonnilpotent element of $S_{i}$. For $k \in Z^{+}, b_{k}-e_{k}=b_{k+1}^{2}$ and so $b_{k}-b_{k+1}$. Thus $0-b_{1}-b_{2}-\cdots$ $-b_{i}-x_{i}$ is a minimal sequence between 0 and $x_{i}$ of length $i$ in both $I_{i}$ and $S$. It now follows easily that $\rho_{1}(S)=\infty, \rho_{1}\left(I_{i}\right)=i$. Since $S, I_{i}$ are $\Gamma$-semigroups, $\rho_{2}(S)=\infty$ and $\rho_{2}\left(I_{i}\right)=i . S$ and each $I_{i}$ are $\delta$-indecomposable since there is a sequence between any two points. It is also clear that if we choose $S_{i}$ 's finite, we obtain finite semigroups of every finite semirank and rank.

Example 4.5. Now we are going to show that Theorem 2.5 need not be true for arbitrary (even finite) semigroups. Let $S, S_{1}, S_{2}$ be as in Example 4.2. Now we 
further assume that there exists a nonzero idempotent $e_{i}$ in $S_{i}$, a nonzero nilpotent element $b_{i} \in S_{i}$ such that $b_{i}^{2}=e_{i} b_{i}=b_{i} e_{i}=0, i=1,2$. (For instance $S_{i}$ could be the Rees-matrix semigroup over the trivial group $\{1\}$ with the $3 \times 3$ identity sandwich matrix and

$$
e_{i}=\left(\begin{array}{lll}
1 & 0 & 0 \\
0 & 0 & 0 \\
0 & 0 & 0
\end{array}\right) \text { and } b_{i}=\left(\begin{array}{lll}
0 & 0 & 0 \\
0 & 0 & 0 \\
0 & 1 & 0
\end{array}\right) \text {. }
$$

Let $T=S \cup\left\{u_{1}, u_{2}, u_{3}, u_{4}\right\}, u_{k} \notin S, k=1,2,3,4, u_{i} \neq u_{j}$ for $i \neq j$. We have

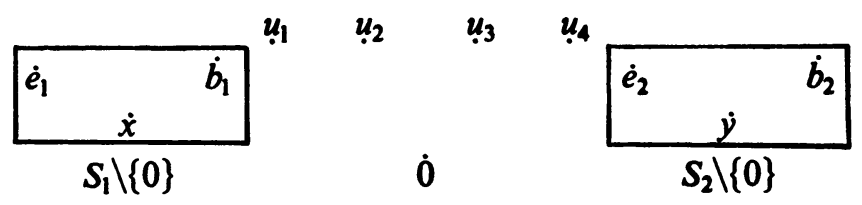

We complete the multiplication table as follows:

(1) $u_{i} u_{j}=0$ for $i \neq j$.

(2) $u_{1}^{2}=e_{1}$.

(3) $u_{2}^{2}=0$.

(4) $u_{3}^{2}=e_{2}$.

(5) $u_{4}^{2}=0$.

(6)

\begin{tabular}{cc}
$x \in S_{1}$ & $y \in S_{2}$ \\
\hline$u_{1} x=e_{1} x$ & $u_{1} y=b_{2} y$ \\
$x u_{1}=x e_{1}$ & $y u_{1}=y b_{2}$ \\
$u_{2} x=b_{1} x$ & $u_{2} y=0$ \\
$x u_{2}=x b_{1}$ & $y u_{2}=0$ \\
$u_{3} x=b_{1} x$ & $u_{3} y=e_{2} y$ \\
$x u_{3}=x b_{1}$ & $y u_{3}=y e_{2}$ \\
$u_{4} x=0$ & $u_{4} y=b_{2} y$ \\
$x u_{4}=0$ & $y u_{4}=y b_{2}$
\end{tabular}

The multiplication intersects when $x=y=0$ but then the values are identically equal to 0 . It can be shown with some effort that $T$ is a semigroup with zero 0 . Furthermore

(i) $u_{1}$ divides every element of $S$.

(ii) $u_{2}$ divides every element of $S_{1}$ but no element of $S_{2} \backslash\{0\}$.

(iii) $u_{3}$ divides every element of $S$.

(iv) $u_{4}$ divides every element of $S_{2}$ but no element of $S_{1} \backslash\{0\}$. Thus, 


$$
\begin{array}{lll}
u_{2} \mid e_{1}=u_{1}^{2}, & u_{1} \mid 0=u_{2}^{2}, & \text { whence } u_{1}-u_{2} . \\
u_{3} \mid e_{1}=u_{1}^{2}, & u_{1} \mid e_{2}=u_{3}^{2}, & \text { whence } u_{1}-u_{3} . \\
u_{4} \mid e_{2}=u_{3}^{2}, & u_{3} \mid 0=u_{4}^{2}, & \text { whence } u_{3}-u_{4} . \\
u_{4} \mid 0=u_{2}^{2}, & u_{2} \mid 0=u_{4}^{2}, & \text { whence } u_{2}-u_{4} .
\end{array}
$$

But,

$$
\begin{array}{ll}
u_{4} \nmid e_{1}=u_{1}^{2} & \text { and so } u_{1} \neq u_{4} . \\
u_{2} \nmid e_{2}=u_{3}^{2} & \text { and so } u_{2} \neq u_{3} .
\end{array}
$$

Thus,

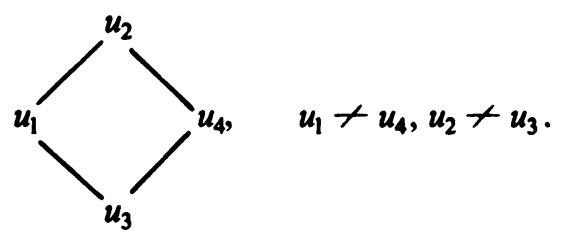

So Theorem 2.5 is not true for $T$. Note that $u_{3} \mid 0=u_{2}^{2}$ and so $u_{3} \rightarrow u_{2}$. A slightly more complicated example can be given where

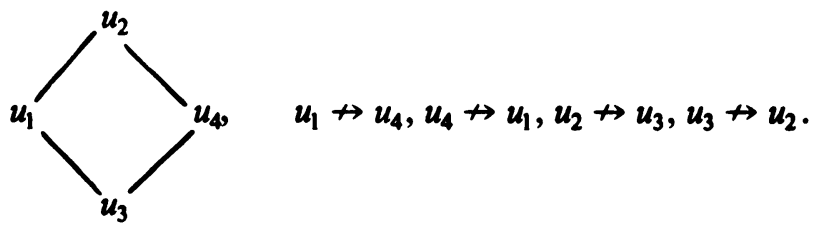

Example 4.6. Let $X$ be a finite set $|X|>2, \beth_{X}$ the full transformation semigroup on $X$. Let $V_{X}=\beth_{X} \mid S_{x}$ where $S_{X}$ is the group of permutations on $X$. Then $V_{X}$ is a prime ideal in $\beth_{X} . V_{X}$ is $\delta$-indecomposable. Moreover, there exists a fixed $a_{0} \in \nu_{X}$ such that for all $c \in \nu_{X}, a_{0}-c$. So $\rho_{1}\left(\nu_{X}\right)=\rho_{2}\left(V_{X}\right)=\rho_{1}\left(\beth_{X}\right)$ $=\rho_{2}\left(\square_{x}\right)=1$. Since $\beth_{x}, V_{x}$ are $\Gamma$-semigroups, Theorem 2.5 is true for these semigroups. As a side remark we mention that $\nu_{X}$ cannot even be decomposed into disjoint union of proper subsemigroups.

Example 4.7. Let $X$ be an infinite set and $\beth_{X}$ the full transformation semigroup on $X$. Then there exists a fixed $a_{0} \in \beth_{X}$ such that for all $c \in \beth_{X}, a_{0}-c$. Furthermore $\beth_{X}$ is a $\Gamma$-semigroup. So $\beth_{X}$ is an $\delta$-indecomposable semigroup of rank and semirank 1. Furthermore Theorem 2.5 holds for $\beth_{x}$. Can $\beth_{x}$ be decomposed into a disjoint union of proper subsemigroups?

Finally the author would like to thank Professor Julian Weissglass for his helpful suggestions. 


\section{REFERENCES}

1. A. H. Clifford, Semigroups admitting relative inverses, Ann. of Math. (2) 42 (1941), 1037-1049. MR 3, 199.

2. A. H. Clifford and G. B. Preston, The algebraic theory of semigroups. Vol. 1, Math. Surveys, no. 7, Amer. Math. Soc., Providence, R.I., 1961. MR 24 \#A2627.

3. M. S. Putcha, Semilattice decompositions of semigroups, Semigroup Forum 6 (1973), 12-34.

4. M. S. Putcha and J. Weissglass, A semilattice decomposition into semigroups having at most one idempotent, Pacific J. Math. 39 (1971), 225-228.

5. T. Tamura, The theory of construction of finite semigroups. I, Osaka Math. J. 8 (1956), 243-261. MR 18, 717.

6. - Another proof of a theorem concerning the greatest semilattice decomposition of a semigroup, Proc. Japan. Acad. 40 (1964), 777-780. MR 31 \#3530.

7.—, On Putcha's theorem concerning semilattice of archimedean semigroups, Semigroup Forum 4 (1972), 83-86.

8.—, Quasi-orders, generalized archimedeaness and semilattice decompositions (to appear).

9. - Note on the greatest semilattice decomposition of semigroups, Semigroup Forum 4 (1972), 255-261.

10. M. Petrich, The maximal semilattice decomposition of a semigroup, Math. Z. 85 (1964), 68-82. MR 29 \#4824.

Department of Mathematics, University of California, Santa Barbara, California 93106

Current address: Department of Mathematics, University of California, Berkeley, California 94720 